\title{
DENSITY AGGREGATION OPERATORS BASED ON THE INTUITIONISTIC TRAPEZOIDAL FUZZY NUMBERS FOR MULTIPLE ATTRIBUTE DECISION MAKING
}

\author{
Peide LIU ${ }^{\mathrm{a}, \mathrm{b}}$, Xiaocun YU \\ ${ }^{a}$ School of Management Science and Engineering, \\ Shandong University of Finance and Economics, 250014 Jinan, China \\ ${ }^{b}$ School of Economics and Management, Civil Aviation University of China, 300300 Tianjin, China
}

Received 14 November 2012; accepted 08 September 2013

\begin{abstract}
With respect to the multiple attribute decision making problems in which the attribute values take the form of the intuitionistic trapezoidal fuzzy numbers, some methods based on density aggregation operators are proposed. Firstly, the definition, expected value and the ranking method of intuitionistic trapezoidal fuzzy numbers are introduced, and the method of calculating density weighted vector is proposed. Then some density aggregation operators based on interval numbers and intuitionistic trapezoidal fuzzy numbers are developed, and a multiple attribute decision making method is presented. Finally an illustrative example is given to verify the developed approach and to demonstrate its practicality and effectiveness.
\end{abstract}

Keywords: density aggregation operator, interval numbers, intuitionistic trapezoidal fuzzy numbers, multiple attribute decision making.

Reference to this paper should be made as follows: Liu, P.; Yu, X. 2013. Density aggregation operators based on the intuitionistic trapezoidal fuzzy numbers for multiple attribute decision making, Technological and Economic Development of Economy 19(Supplement 1): S454-S470.

JEL Classification: C60, D81.

\section{Introduction}

Multi-attribute decision making (MADM) has a wide range of applications, such as personal assessment, product evaluation, employee performance evaluation, economic evaluation, investment decision making, risk assessment, etc. Due to the complexity and uncertainty

Corresponding author Peide Liu

E-mail: peide.liu@gmail.com 
of the decision-making environment, we need consider various aspects in the evaluation process so as to make a scientific and rational decision.

In recent years, Research on the MADM problems with the intuitionistic fuzzy information has made a lot of achievements (Atanassov 1989; Liu 2009, 2013a, b, c; Liu, Jin 2012; Liu et al. 2012; Xu, Yager 2006; Xu 2007; Yu 2013). Atanassov (1986, 1989) proposed the intuitionistic fuzzy set (IFS) which is the generalization of the concept of fuzzy set. Xu and Yager (2006), Xu (2007) proposed some aggregation operators with intuitionistic fuzzy information. Yu (2013) proposed some intuitionistic fuzzy prioritized operators and applied them to multi-criteria group decision making. Razavi Hajiagha et al. (2013) proposed a complex proportional assessment method for group decision making in an interval-valued intuitionistic fuzzy environment. Zhang and Liu (2010) proposed the triangular intuitionistic fuzzy number which used the triangular fuzzy number to denote the membership degree and the non-membership degree, and then the weighted arithmetic average operator was defined. Further, Wang (2008), Wang and Zhang (2008) gave the definition of intuitionistic trapezoidal fuzzy number, and defined the expected values of intuitionistic trapezoidal fuzzy number and proposed a decision method based on intuitionistic trapezoidal fuzzy number. Wang and Zhang (2009a) proposed the Hamming distance between intuitionistic trapezoidal fuzzy numbers and intuitionistic trapezoidal fuzzy weighted arithmetic averaging (ITFWAA) operator. Wang and Zhang (2009b) proposed some aggregation operators, including intuitionistic trapezoidal fuzzy weighted arithmetic averaging operator and weighted geometric averaging operator. Du and Liu (2011) proposed the extended VIKOR method based on the intuitionistic trapezoidal fuzzy numbers. Zhang et al. (2013) proposed the grey relational projection method for multi-attribute decision making based on intuitionistic trapezoidal fuzzy numbers. Wan (2013) proposed some power average operators of trapezoidal intuitionistic fuzzy numbers and application to multi-attribute group decision making.

WAA (or WGA) operator can only weight the information by importance of all attributes, and OWA operator weights the ordered positions of all attributes. But the two weighted methods don't consider the distribution of density degree of the attribute values. However, density of decision making information is an important index for decision making. In a set of data, the high concentration of data reflects the high consistency of information; on the contrary, the high dispersion of the data reflects the low consistency of information. According to the preferences of decision makers, we could pay attention to the high consistency of information (emphasis on group opinion) or to the low consistency of information (emphasis on individual opinions). Based on the distribution of density degree of attributes, Yi et al. (2007) proposed the density-weighted averaging (DWA) operator to aggregate attribute values which are crisp numbers, then Hou and Guo (2008), Li et al. (2012) proposed the density-weighted averaging (DWA) operators to aggregate interval numbers.

The proposed density aggregation operators above are only used for crisp numbers or interval numbers. In this paper, we will extend them to the intuitionistic trapezoidal fuzzy numbers, and propose a decision making method. 


\section{Intuitionistic trapezoidal fuzzy number}

\subsection{The definition and operational laws of intuitionistic trapezoidal fuzzy numbers}

Definition 1 (Wang, Zhang 2009a): let $\tilde{a}$ be an intuitionistic trapezoidal fuzzy number in the set $R$ of real numbers, its membership function is given by:

$$
\mu_{\tilde{a}}(x)= \begin{cases}\frac{x-a}{b-a} \mu_{\tilde{a}}, & a \leq x<b \\ \mu_{\tilde{a}}, & b \leq x \leq c \\ \frac{d-x}{d-c} \mu_{\tilde{a}}, & c<x \leq d \\ 0, & \text { otherwise }\end{cases}
$$

and its non-membership function is given by:

$$
v_{\tilde{a}}(x)=\left\{\begin{array}{ll}
\frac{(b-x)+v_{\tilde{a}}\left(x-a_{1}\right)}{b-a_{1}}, & a_{1} \leq x<b \\
v_{\tilde{a}}, & b \leq x \leq c \\
\frac{(x-c)+v_{\tilde{a}}\left(d_{1}-x\right)}{d_{1}-c}, & c<x \leq d_{1} \\
0, & \text { otherwise }
\end{array},\right.
$$

where: $0 \leq \mu_{\tilde{a}} \leq 1, \quad 0 \leq v_{\tilde{a}} \leq 1, \quad 0 \leq \mu_{\tilde{a}}+v_{\tilde{a}} \leq 1$ and $a, a_{1}, b, c, d, d_{1} \in R$. Therefore, $\tilde{a}=\left\langle\left([a, b, c, d] ; \mu_{\tilde{a}}\right),\left(\left[a_{1}, b, c, d_{1}\right] ; v_{\tilde{a}}\right)\right\rangle$ is called the intuitionistic trapezoidal fuzzy number (ITFN). If $\mu_{\tilde{a}}=1, v_{\tilde{a}}=0$, then $\tilde{a}$ is reduced to a traditional trapezoidal fuzzy number; if $b=c$, then $\tilde{a}$ is reduced to an intuitionistic triangular fuzzy number. Generally, in the intuitionistic trapezoidal fuzzy number $\tilde{a}$, there is $[a, b, c, d]=\left[a_{1}, b, c, d_{1}\right]$, and then $\tilde{a}$ is denoted as $\tilde{a}=\left([a, b, c, d] ; \mu_{\tilde{a}}, v_{\tilde{a}}\right)$. In this paper, if there is no special statement, we will adopt the intuitionistic trapezoidal fuzzy number $\tilde{a}=\left([a, b, c, d] ; \mu_{\tilde{a}}, v_{\tilde{a}}\right)$. For each $x \in R$, if $\pi_{\tilde{a}}(x)=1-\mu_{\tilde{a}}(x)-v_{\tilde{a}}(x), \pi_{\tilde{a}}(x)$ is called the degree of indeterminacy of $x$ to $\tilde{a}$. The smaller value of $\pi_{\tilde{a}}(x)$ is, the more certain of $x$ to $\tilde{a}$ is.

Some operational laws on intuitionistic trapezoidal fuzzy numbers are shown as follows:

Definition 2 (Wang, Zhang 2009a): Let $\tilde{a}_{1}=\left(\left[a_{1}, b_{1}, c_{1}, d_{1}\right] ; \mu_{\tilde{a}_{1}}, v_{\tilde{a}_{1}}\right)$ and $\tilde{a}_{2}=\left(\left[a_{2}, b_{2}, c_{2}, d_{2}\right] ; \mu_{\tilde{a}_{2}}, v_{\tilde{a}_{2}}\right)$ be two intuitionistic trapezoidal fuzzy numbers, and $\lambda \geq 0$, we have:

$$
\begin{gathered}
\tilde{a}_{1}+\tilde{a}_{2}=\left(\left[a_{1}+a_{2}, b_{1}+b_{2}, c_{1}+c_{2}, d_{1}+d_{2}\right] ; \mu_{\tilde{a}_{1}}+\mu_{\tilde{a}_{2}}-\mu_{\tilde{a}_{1}} \mu_{\tilde{a}_{2}}, v_{\tilde{a}_{1}} v_{\tilde{a}_{2}}\right) ; \\
\tilde{a}_{1} \otimes \tilde{a}_{2}=\left(\left[a_{1} a_{2}, b_{1} b_{2}, c_{1} c_{2}, d_{1} d_{2}\right] ; \mu_{\tilde{a}_{1}} \mu_{\tilde{a}_{2}}, v_{\tilde{a}_{1}}+v_{\tilde{a}_{2}}-v_{\tilde{a}_{1}} v_{\tilde{a}_{2}}\right) ; \\
\lambda \tilde{a}_{1}=\left(\left[\lambda a_{1}, \lambda b_{1}, \lambda c_{1}, \lambda d_{1}\right] ; 1-\left(1-\mu_{\tilde{a}_{1}}\right)^{\lambda}, v_{\tilde{a}_{1}}^{\lambda}\right) ; \\
\tilde{a}_{1}^{\lambda}=\left(\left[a_{1}^{\lambda}, b_{1}^{\lambda} c_{1}^{\lambda} d_{1}^{\lambda}\right] ; \mu_{\tilde{a}_{1}}^{\lambda}, 1-\left(1-v_{\tilde{a}_{1}}\right)^{\lambda}\right) .
\end{gathered}
$$




\subsection{The expected value of an intuitionistic trapezoidal fuzzy number}

For an intuitionistic trapezoidal fuzzy number $\tilde{a}$, we can define $f_{\tilde{a}}^{L}(x)=\frac{x-a}{b-a} \mu_{\tilde{a}}(x)$, $f_{\tilde{a}}^{R}(x)=\frac{d-x}{d-c} \mu_{\tilde{a}}(x)$. According to the characteristics of these two functions, we know that $f_{\tilde{a}}^{L}(x)$ is a monotone increasing function on its interval $[a, b]$, and $f_{\tilde{a}}^{R}(x)$ is a monotone decreasing function on its interval $[c, d]$, and then their inverse functions are respectively given as follows (Wang, Zhang 2008):

$$
\begin{aligned}
& p_{\tilde{a}}^{L}(y)=a+\frac{y}{\mu_{\tilde{a}}}(b-a), y \in\left(0, \mu_{\tilde{a}}\right] ; \\
& p_{\tilde{a}}^{R}(y)=d+\frac{y}{\mu_{\tilde{a}}}(c-d), y \in\left(0, \mu_{\tilde{a}}\right] .
\end{aligned}
$$

For the intuitionistic trapezoidal fuzzy number $\tilde{a}$, the confidence interval of the trapezoidal fuzzy number $[a, b, c, d]$ is $\left[\mu_{\tilde{a}}, 1-v_{\tilde{a}}\right]$ (Wang, Zhang 2008).

Definition 3 (Wang, Zhang 2008):

$$
I_{\lambda}^{L}(\tilde{a})=\int_{0}^{\mu_{\tilde{a}}}\left\{(1-\lambda)\left[a+\frac{y}{\mu_{\tilde{a}}}(b-a)\right]+\lambda\left[d+\frac{y}{\mu_{\tilde{a}}}(c-d)\right]\right\} d y,
$$

is called the minimum expected value for the intuitionistic trapezoidal fuzzy number $\tilde{a}$, where, $\lambda \in[0,1]$ denotes the risk preferences of decision makers. If $\lambda>0.5$, decision makers tend to risk- pursuit; if $\lambda<0.5$, decision makers tend to risk-aversion, if $\lambda=0.5$, decision makers tend to risk-neutrality. Then we can get:

$$
I_{\lambda}^{L}(\tilde{a})=\frac{\mu_{\tilde{a}}}{2}[(1-\lambda)(a+b)+\lambda(c+d)] .
$$

Definition 4 (Wang, Zhang 2008):

$$
I_{\lambda}^{R}(\tilde{a})=\int_{0}^{1-v_{\tilde{a}}}\left\{(1-\lambda)\left[a+\frac{y}{1-v_{\tilde{a}}}(b-a)\right]+\lambda\left[d+\frac{y}{1-v_{\tilde{a}}}(c-d)\right]\right\} d y,
$$

is called the maximum expected value for the intuitionistic trapezoidal fuzzy number of $\tilde{a}$. Similarly, $\lambda \in[0,1]$ denotes the risk preferences of decision makers. If $\lambda>0.5$, decision makers tend to risk- pursuit; if $\lambda<0.5$, decision makers tend to risk-aversion, if $\lambda=0.5$, decision makers tend to risk-neutrality. Then we can get:

$$
I_{\lambda}^{R}(\tilde{a})=\frac{1-v_{\tilde{a}}}{2}[(1-\lambda)(a+b)+\lambda(c+d)] .
$$

Therefore, we get the expected value interval of intuitionistic trapezoidal fuzzy number of $\tilde{a}$ is $\left[I_{\lambda}^{L}(\tilde{a}), I_{\lambda}^{R}(\tilde{a})\right]$.

For two intuitionistic trapezoidal fuzzy numbers, we could rank them by their expected value intervals according to the ranking method of the interval numbers. 


\section{Density weighting vector}

\subsection{The clustering method}

Yi et al. (2007) proposed a clustering method for point data element, which is called the ordered incremental segmentation method, but it is unable to handle multidimensional data elements, such as vector and matrix. Further, Yi and Guo (2010) proposed a new general method to cluster the data elements for point, vector and matrix.

Definition 5 (Yi, Guo 2010): Let $a_{i}$ and $a_{j}(i, j \in R, i \neq j)$ be any two elements of $A$, $A=\left\{a_{1}, a_{2}, \ldots \cdots, a_{n}\right\}$, and $N=(1,2, \cdots, n)$, then the distance between $a_{i}$ and $a_{j}$ is given by: If $a_{i}$ and $a_{j}$ are point elements, then $d_{i j}=\left|a_{i}-a_{j}\right|$.

If $a_{i}$ and $a_{j}$ are two vectors, and $a_{k}=\left(x_{1 k}, x_{2 k}, \cdots, x_{s k}\right)^{\mathrm{T}}, k \in N$,

then

$$
d_{i j}=\sqrt{\sum_{l=1}^{s}\left(x_{l i}-x_{l j}\right)^{2}} .
$$

If $a_{i}$ and $a_{j}$ are two matrixes, $a_{k}=\left[x_{l t}^{(k)}\right]_{s \times p} k \in N$,

then

$$
d_{i j}=\sqrt{\sum_{l=1}^{s} \sum_{t=1}^{p}\left(x_{l t}^{(i)}-x_{l t}^{(j)}\right)^{2}} .
$$

Definition 6 (Yi, Guo 2010): let $M=(1,2, \cdots, m)$ and $A_{t}(t \in M)$ be any data subset of $A_{1}, A_{2}, \ldots, A_{m}$, and the number of its elements is $k_{t}$, then the distance between $A_{i}$ and $A_{j}(i, j \in M, i \neq j)$ is given by:

$$
\delta\left(A_{i}, A_{j}\right)=\frac{1}{k_{i} k_{j}} \sum_{g=1}^{k_{j}} \sum_{h=1}^{k_{i}} d_{h g},
$$

where: $d_{h g}\left(h=1,2, \cdots, k_{i} ; g=1,2, \cdots, k_{j}\right)$ denotes the distance between the hth element in $A_{i}$ and the gth element in $A_{j}$.

Based on (13)-(16), we can compute the distance between elements or between the data subsets respectively, and then complete the process of cluster grouping of data elements. For the detailed steps, please refer to Yu and Fan (2003).

\subsection{Determining the density weighting vectors}

Yi and Guo (2010) proposed a method for balancing the weight vector of "scale" and "function", and its form is given by:

$$
\xi_{i}=\rho \omega_{i}^{s}+v \omega_{i}^{f}(i \in M),
$$

where: $\xi_{i}$ is the ith component of $\xi=\left(\xi_{1}, \xi_{2}, \ldots, \xi_{m}\right)$ corresponding to the data subset $A_{i}$; $\omega^{s}=\left(\omega_{1}^{s}, \omega_{2}^{s}, \cdots, \omega_{m}^{s}\right)$ is the "scale" weight of $\xi$, and $\sum_{i=1}^{m} \omega_{i}^{s}=1 ; \omega^{f}=\left(\omega_{1}^{f}, \omega_{2}^{f}, \cdots, \omega_{m}^{f}\right)$ is the "function" weight of $\xi$, and $\sum_{i=1}^{m} \omega_{i}^{f}=1 ; \rho$ and $v$ are the adjustment factors, and meet
$\rho, v \in[0,1], \rho+v=1$. 
According to Yi and Guo (2010), if $\omega_{1}, \omega_{2}, \cdots, \omega_{n}$ are the "function" weights of $a_{1}, a_{2}, \cdots, a_{n}$, respectively, and $\omega_{k} \geq 0, \sum_{k \in N} \omega_{k}=1$, then:

$$
\omega_{i}^{f}=\sum_{k \in N, a_{k} \in A_{i}} \omega_{k},(i \in M) .
$$

Based on the "scale" connotation of $\omega^{s}=\left(\omega_{1}^{s}, \omega_{2}^{s}, \cdots, \omega_{m}^{s}\right)$, we can confirm that $\omega^{s}$ is a function on the $k_{i}\left(k_{i}\right.$ is the number of data subset $\left.A_{i}(i \in M)\right)$, and we can specify an exponential function:

$$
f^{ \pm}(\gamma)=a+b e^{ \pm(c \gamma)}
$$

where: $\gamma \in(0,1), a, b$ and $c$ are undetermined coefficients, and $b, c>0$.

Definition 7 (Yi, Guo 2010): $f^{+}(\gamma)=a+b e^{(c \gamma)}$ is called the positive gain function of "scale"; $f^{-}(\gamma)=a+b e^{-c \gamma}$ is called the negative gain function of "scale". $f^{ \pm}(\gamma)$ satisfies the following conditions:

(1) Zero condition: $f^{+}(\gamma)=0$ or $f^{-}(\gamma)=0$;

(2) Normalization condition: $\sum_{i \in M} f^{ \pm}\left(k_{i} / n\right)=1$;

(3) Ratio condition: $f^{ \pm}\left(k_{i} / n\right) / f^{ \pm}\left(k_{j} / n\right)=\eta ; i, j \in M, i \neq j$.

where: $\eta(\eta>0)$ is the ratio which is determined by the maker's judgment, and it is used to provide an entry point for the maker's preference judgment. If $k_{i}>k_{j}$, we can allow $\eta>1$ in the function of $f^{+}(\gamma)$ and $\eta<1$ in the function of $f^{-}(\gamma)$. If this condition is not needed, we can allow $c=1$.

The above conditions are converted to the following formulas.

$$
\left\{\begin{array}{l}
a+b=0 \\
m a+\sum_{l=1}^{m} b e^{ \pm\left(c k_{l} / n\right)}=1 \\
\left(a+b e^{ \pm c k_{i} / n}\right) /\left(a+b e^{ \pm c k_{j} / n}\right)=\eta
\end{array}\right.
$$

where: " \pm " can get " + " or " - " which is determined by the characteristics of function. By solving Eq. (20), we can get parameters $a, b$ and $c$, and get the precise form of function $f^{ \pm}(\gamma)$. Then let:

$$
\omega_{i}^{s}=\omega_{i}^{s}\left(k_{i} / n\right)=f^{ \pm}\left(k_{i} / n\right)(i \in M),
$$

to calculate $\omega_{i}^{s}$. Based on condition (2), we can get $\sum_{i \in M} \omega_{i}^{s}=1$.

After getting the values of $\omega_{i}^{f}$ and $\omega_{i}^{s}$, for Eq. (17), if $\omega^{f} \neq \omega^{s}$ (this is a normal condition), the values of $\rho$ and $v$ will be two parameters to be determined to calculate $\xi_{i}$. The following, we will give some methods to determine their values. 
Definition 8 (Yi, Guo 2010): The measuring degree of "group similarity" of the density weighting vector $\xi=\left(\xi_{1}, \xi_{2}, \cdots, \xi_{m}\right)$ can be defined as follows:

$$
T s(\xi)=\frac{1}{m-1} \sum_{i=1}^{m}(m-i) \xi_{i}
$$

and the measuring degree of "group differences" can be given by:

$$
T e(\xi)=1-T s(\xi) .
$$

It is easily proved that $T s(\xi), T e(\xi) \in[0,1]$. If $T s(\xi)>0.5$ (when the positive gain function of "scale" is chosen), it shows that decision makers pay more attention to "large group consensus" of groups; contrarily, if $T s(\xi)<0.5$ (when the negative gain function of scale is chosen), it shows that decision makers pay more attention to "small group consensus" of groups.

Definition 9 (Yi, Guo 2010): The measure of entropy of density weighting vector $\xi=\left(\xi_{1}, \xi_{2}, \cdots, \xi_{m}\right)$ is given by:

$$
\operatorname{En}(\xi)=\frac{1}{\ln m} \sum_{i=1}^{m} \xi_{i} \ln \xi_{i}
$$

$\operatorname{En}(\xi) \in[0,1]$ denotes the information amount of the density weighting vector $\xi$ and reflects the balanced state between component values.

Based on the Definitions 8 and 9, two ways are given to calculate the values of $\rho$ and $v$.

(1) Preference coefficients method (also be called the subjective method).

Suppose that decision maker gives a preference level value $\pi$ on the degree of "group similarity" in advance, based on Eqs. (17) and (19), we can get:

$$
\frac{1}{m-1} \sum_{i=1}^{m}(m-i)\left(\rho \omega_{i}^{s}+v \omega_{i}^{f}\right)=\pi \text {. }
$$

Theorem 1 (Yi, Guo 2010): If $\pi$ denotes the measuring degree of "group similarity" $\operatorname{Ts}(\xi)$, we can get:

$$
\left\{\begin{array}{l}
\rho=\left(m-(m-1) \pi-\sum_{i=1}^{m} i \omega_{i}^{f}\right) / \sum_{i=1}^{m} i\left(\omega_{i}^{s}-\omega_{i}^{f}\right) \\
\left.\mathrm{u}=(-m+(m-1) \pi)+\sum_{i=1}^{m} i \omega_{i}^{s}\right) / \sum_{i=1}^{m} i\left(\omega_{i}^{s}-\omega_{i}^{f}\right)
\end{array} .\right.
$$
Combined Eq. (25) with known conditions that $\sum_{i=1}^{m} \omega_{i}^{s}=1, \sum_{i=1}^{m} \omega_{i}^{f}=1, \rho+v=1$, we can
prove Theorem 1 .

The valid range of preference value on the degree of "group similarity" can be set as follows (Yi, Guo 2010)

$$
\pi \in\left[\min \left(\frac{1}{m-1} \sum_{i=1}^{m}(m-i) \omega_{i}^{f}, \frac{1}{m-1} \sum_{i=1}^{m}(m-i) \omega_{i}^{s}\right), \max \left(\frac{1}{m-1} \sum_{i=1}^{m}(m-i) \omega_{i}^{f}, \frac{1}{m-1} \sum_{i=1}^{m}(m-i) \omega_{i}^{s}\right)\right] .
$$

Eq. (25) can be seen as a linear function $\pi$ with respect to $\rho$, and then we can calculate the range of the value of $\pi(\rho)$ on $[0,1]$ by considering the positive or negative value of $\frac{d \pi}{d \rho}$. 
(2) Maximum entropy method (also be called objective method).

From the properties of entropy, the maximum entropy is the best balance of components' difference of density weighted vector $\xi=\left(\xi_{1}, \xi_{2}, \cdots, \xi_{m}\right)$. So we can select the values of $\rho$ and $v$ by maximizing the entropy value $\operatorname{En}(\rho)$. Then we will put the condition $\rho=1-v$ and Eq. (17) into Eq. (24), and get:

$$
\operatorname{En}(\rho)=-\frac{1}{\ln m} \sum_{i=1}^{m}\left(\rho \omega_{i}^{s}+(1-\rho) \omega_{i}^{f}\right) \bullet \ln \sum_{i=1}^{m}\left(\rho \omega_{i}^{s}+(1-\rho) \omega_{i}^{f}\right) .
$$

Then we can calculate the first and second derivatives of $\operatorname{En}(\rho)$, and it is easy to know that $\operatorname{En}^{\prime \prime}(\rho)>0$ for all $\rho$, i.e. $\operatorname{En}(\rho)$ is a strict concave function. So, the condition of maximizing $\operatorname{En}(\rho)$ is shown as follows:

$$
\rho=\left\{\begin{array}{cc}
\rho_{0} & \rho_{0} \in[0,1] \\
0 & \rho_{0}<0 \\
1 & \rho_{0}>1
\end{array},\right.
$$

where: $\rho_{0}$ is the point which make that $\operatorname{En}^{\prime}(\rho)=0$.

\section{Some density aggregation operators based on intuitionistic trapezoidal fuzzy numbers}

Definition 10: Let $\tilde{a}_{j}=\left[\underline{a}_{j}, \bar{a}_{j}\right]$ be a collection of interval numbers, and $\omega=\left(\omega_{1}, \omega_{2}, \cdots, \omega_{n}\right)^{T}$ be the weight vector of $\tilde{a}_{j}(j=1,2, \cdots, n)$, where $\omega_{j} \geq 0, j=1,2, \cdots, n$ and $\sum_{j=1}^{n} \omega_{j}=1$, then we can define an interval density weighted average (IDWA ) operator as follows:

$$
\operatorname{IDWA}\left(\tilde{a}_{1}, \tilde{a}_{2}, \cdots, \tilde{a}_{n}\right)=\sum_{i=1}^{m} \xi_{i} \bullet \operatorname{WAA}\left(\mathrm{A}_{\mathrm{i}}\right)=\sum_{i=1}^{m} \xi_{i}\left(\sum_{j=1}^{k_{i}} \omega_{j}^{(i)} \tilde{a}_{j}^{(i)}\right),
$$

where: $A_{i}=\left\{\tilde{a}_{j}^{(i)} \mid i=1,2, \cdots, m ; j=1,2, \cdots, k_{i}\right\}$, and $\sum_{i=1}^{m} k_{i}=n . A_{1}, A_{2}, \cdots, A_{m}$ are $m$ clustering classes, and $\tilde{a}_{j}^{(i)}$ is the data in class $A_{\mathrm{i}} ; \xi=\left(\xi_{1}, \xi_{2}, \cdots, \xi_{m}\right)$ is the density weighted vector; $\omega_{i}=\left(\omega_{1}^{(i)}, \omega_{2}^{(i)}, \ldots, \omega_{k_{i}}^{(i)}\right)$ is the weight of $A_{i}=\left(\tilde{a}_{1}^{(i)}, \tilde{a}_{2}^{(i)}, \ldots, \tilde{a}_{k_{i}}^{(i)}\right)$, then IDWA is called the interval density weighted average operator, also called IDWA operator.

Further, according to the operational rules of interval numbers, we can get:

$$
\operatorname{IDWA}\left(\tilde{a}_{1}, \tilde{a}_{2}, \cdots, \tilde{a}_{n}\right)=\left[\sum_{i=1}^{m} \xi_{i}\left(\sum_{j=1}^{k_{i}} \omega_{j}^{(i)} \underline{a}_{j}^{(i)}\right), \sum_{i=1}^{m} \xi_{i}\left(\sum_{j=1}^{k_{i}} \omega_{j}^{(i)} \bar{a}_{j}^{(i)}\right)\right] .
$$

Definition 11: Let $\tilde{a}_{j}=\left[\underline{a}_{j}, \bar{a}_{j}\right]$ be a collection of interval numbers, we can define an interval density ordered weighted average ( IDOWA ) operator as follows:

$$
\operatorname{IDOWA}\left(\tilde{a}_{1}, \tilde{a}_{2}, \cdots, \tilde{a}_{n}\right)=\sum_{i=1}^{m} \xi_{i} \bullet \operatorname{OWA}\left(\mathrm{A}_{\mathrm{i}}\right)=\sum_{i=1}^{m} \xi_{i}\left(\sum_{j=1}^{k_{i}} w_{j}^{(i)} \tilde{b}_{j}^{(i)}\right),
$$


where: $\mathrm{A}_{i}=\left\{\tilde{a}_{j}^{(i)} \mid i=1,2, \cdots, m ; j=1,2, \cdots, k_{i}\right\}, \mathrm{A}_{1}, \mathrm{~A}_{2}, \cdots, \mathrm{A}_{\mathrm{m}}$ are $m$ clustering classes, and $\sum_{i=1}^{m} k_{i}=n . \tilde{b}_{j}^{(i)}$ is the $j$ th largest element in $\left(\tilde{a}_{1}^{(i)}, \tilde{a}_{2}^{(i)}, \cdots, \tilde{a}_{k_{i}}^{(i)}\right)$, and $\xi=\left(\xi_{1}, \xi_{2}, \cdots, \xi_{m}\right)$ is the density weighted vector; $w_{i}=\left(w_{1}^{(i)}, w_{2}^{(i)}, \ldots, w_{k_{i}}^{(i)}\right)$ is an associated weight vector with OWA, and satisfying $\sum_{j=1}^{k_{i}} w_{j}^{(i)}=1, w_{j}^{(i)}>0$. Then IDOWA is called the interval density ordered weighted average operator, also called IDOWA operator.

Further, according to the operational rules of interval numbers, we can get:

$$
\operatorname{IDOWA}\left(\tilde{a}_{1}, \tilde{a}_{2}, \cdots, \tilde{a}_{n}\right)=\left[\sum_{i=1}^{m} \xi_{i}\left(\sum_{j=1}^{k_{i}} w_{j}^{(i)} \underline{b}_{j}^{(i)}\right), \sum_{i=1}^{m} \xi_{i}\left(\sum_{j=1}^{k_{i}} w_{j}^{(i)} \bar{b}_{j}^{(i)}\right)\right] \text {. }
$$

From Definitions 10 and 11, we know that the IDWA operator weights the interval numbers while the IDOWA operator weights the ordered positions of the interval numbers instead of weighting the arguments themselves. Therefore, weights in both the IDWA operator and the IDOWA operator represent different aspects. However, these two operators consider only one of them. To overcome this drawback, in the following we shall propose an interval density hybrid weighted average (IDHWA) operator.

Definition 12: Let $\tilde{a}_{j}=\left[\underline{a}_{j}, \bar{a}_{j}\right]$ be a collection of interval numbers, and $\omega=\left(\omega_{1}, \omega_{2}, \cdots, \omega_{n}\right)^{T}$ be the weight vector of $\tilde{a}_{j}(j=1,2, \cdots, n)$, where $\omega_{j} \geq 0, j=1,2, \cdots, n$ and $\sum_{j=1}^{n} \omega_{j}=1$. Then we can define an interval density hybrid weighted average (IDHWA) operator as follows:

$$
\operatorname{IDHWA}\left(\tilde{a}_{1}, \tilde{a}_{2}, \cdots, \tilde{a}_{n}\right)=\sum_{i=1}^{m} \xi_{i} \bullet \operatorname{HWA}\left(\mathrm{A}_{\mathrm{i}}\right)=\sum_{i=1}^{m} \xi_{i}\left(\sum_{j=1}^{k_{i}} w_{j}^{(i)} \tilde{b}_{j}^{(i)}\right)
$$

where: $\mathrm{A}_{i}=\left\{\tilde{a}_{j}^{(i)} \mid i=1,2, \cdots, m ; j=1,2, \cdots, k_{i}\right\}, \mathrm{A}_{1}, \mathrm{~A}_{2}, \cdots, \mathrm{A}_{\mathrm{m}}$ are $m$ clustering classes, and $\sum_{i=1}^{m} k_{i}=n . \tilde{b}_{j}^{(i)}$ is the $j$ th largest element in $\left(n \omega_{1}^{(i)} \tilde{a}_{1}^{(i)}, n \omega_{2}^{(i)} \tilde{a}_{2}^{(i)}, \cdots, n \omega_{k_{i}}^{(i)} \tilde{a}_{k_{i}}^{(i)}\right)$, and $\xi=\left(\xi_{1}, \xi_{2}, \ldots, \xi_{m}\right)$ is the density weighted vector; $w_{i}=\left(w_{1}^{(i)}, w_{2}^{(i)}, \ldots, w_{k_{i}}^{(i)}\right)$ is an associated weight vector with HWA, and satisfying $\sum_{j=1}^{k_{i}} w_{j}^{(i)}=1, w_{j}^{(i)}>0$, then IDHWA is called the interval density hybrid weighted average operator, also called IDHWA operator.

Similarly, we can get:

$$
\operatorname{IDHWA}\left(\tilde{a}_{1}, \tilde{a}_{2}, \cdots, \tilde{a}_{n}\right)=\left[\sum_{i=1}^{m} \xi_{i}\left(\sum_{j=1}^{k_{i}} w_{j}^{(i)} \underline{b}_{j}^{(i)}\right), \sum_{i=1}^{m} \xi_{i}\left(\sum_{j=1}^{k_{i}} w_{j}^{(i)} \bar{b}_{j}^{(i)}\right)\right] .
$$

The following, we can define some density aggregation operator based on intuitionistic trapezoidal fuzzy numbers. 
Definition 13: Let $\tilde{a}_{j}=\left(\left[a_{j}, b_{j}, c_{j}, d_{j}\right] ; \mu_{j}, v_{j}\right)$ be a collection of intuitionistic trapezoidal fuzzy numbers, and $\omega=\left(\omega_{1}, \omega_{2}, \cdots, \omega_{n}\right)^{T}$ be the weight vector of $\tilde{a}_{j}(j=1,2, \cdots, n)$, where $\omega_{j} \geq 0, j=1,2, \cdots, n$ and $\sum_{j=1}^{n} \omega_{j}=1$, then we can define an intuitionistic trapezoidal fuzzy density weighted average ( ITFDWA ) operator as follows:

$$
\operatorname{ITFDWA}\left(\tilde{a}_{1}, \tilde{a}_{2}, \cdots, \tilde{a}_{n}\right)=\sum_{i=1}^{m} \xi_{i} \bullet \operatorname{WAA}\left(A_{\mathrm{i}}\right)=\sum_{i=1}^{m} \xi_{i}\left(\sum_{j=1}^{k_{i}} \omega_{j}^{(i)} \tilde{a}_{j}^{(i)}\right)
$$

where: $A_{i}=\left\{\tilde{a}_{j}^{(i)} \mid i=1,2, \cdots, m ; j=1,2, \cdots, k_{i}\right\}$, and $\sum_{i=1}^{m} k_{i}=n . A_{1}, A_{2}, \cdots, A_{\mathrm{m}}$ are $m$ clustering classes, and $\tilde{a}_{j}^{(i)}$ is the data of $A_{\mathrm{i}} ; \xi=\left(\xi_{1}, \xi_{2}, \cdots, \xi_{m}\right)$ is the density weighted vector; $\omega_{i}=\left(\omega_{1}^{(i)}, \omega_{2}^{(i)}, \ldots, \omega_{k_{i}}^{(i)}\right)$ is the weight of $A_{i}=\left(\tilde{a}_{1}^{(i)}, \tilde{a}_{2}^{(i)}, \ldots, \tilde{a}_{k_{i}}^{(i)}\right)$, then ITFDWA is called the intuitionistic trapezoidal fuzzy density weighted average operator.

Further, according to the operational rules of intuitionistic trapezoidal fuzzy numbers, we can get:

$$
\begin{gathered}
\operatorname{ITFDWA}\left(\tilde{a}_{1}, \tilde{a}_{2}, \cdots, \tilde{a}_{n}\right)=\left(\left[\sum_{i=1}^{m} \xi_{i} \sum_{j=1}^{k_{i}} \omega_{j}^{(i)} a_{j}^{(i)}, \sum_{i=1}^{m} \xi_{i} \sum_{j=1}^{k_{i}} \omega_{j}^{(i)} b_{j}^{(i)}, \sum_{i=1}^{m} \xi_{i} \sum_{j=1}^{k_{i}} \omega_{j}^{(i)} c_{j}^{(i)}, \sum_{i=1}^{m} \xi_{i} \sum_{j=1}^{k_{i}} \omega_{j}^{(i)} d_{j}^{(i)},\right] ;\right. \\
\left.1-\prod_{i=1}^{m}\left(1-\left(1-\prod_{j=1}^{k_{i}}\left(1-\mu_{\tilde{a}_{j}^{(i)}}\right)^{\omega_{j}^{(i)}}\right)\right)^{\xi_{i}}, \prod_{i=1}^{m}\left(\prod_{j=1}^{k_{i}}\left(v_{\tilde{a}_{j}^{(i)}}\right)^{\omega_{j}^{(i)}}\right)^{\xi_{i}}\right) .
\end{gathered}
$$

Definition 14: Let $\tilde{a}_{j}=\left(\left[a_{j}, b_{j}, c_{j}, d_{j}\right] ; \mu_{j}, v_{j}\right)$ be a collection of intuitionistic trapezoidal fuzzy numbers, we can define an intuitionistic trapezoidal fuzzy density ordered weighted average ( ITFDOWA ) operator as follows:

$$
\operatorname{ITFDOWA}\left(\tilde{a}_{1}, \tilde{a}_{2}, \cdots, \tilde{a}_{n}\right)=\sum_{i=1}^{m} \xi_{i} \bullet \operatorname{OWA}\left(\mathrm{A}_{\mathrm{i}}\right)=\sum_{i=1}^{m} \xi_{i}\left(\sum_{j=1}^{k_{i}} w_{j}^{(i)} \tilde{a}_{\sigma(j)}^{(i)}\right)
$$

where: $\mathrm{A}_{i}=\left\{\tilde{a}_{j}^{(i)} \mid i=1,2, \cdots, m ; j=1,2, \cdots, k_{i}\right\}$, and $\sum_{i=1}^{m} k_{i}=n . \mathrm{A}_{1}, \mathrm{~A}_{2}, \ldots, \mathrm{A}_{m}$ are $m$ clustering classes, and $\tilde{a}_{j}^{(i)}$ is the data of $A_{i} ; \xi=\left(\xi_{1}, \xi_{2}, \cdots, \xi_{m}\right)$ is the density weighted vector; and $w_{i}=\left(w_{1}^{(i)}, w_{2}^{(i)}, \ldots, w_{k_{i}}^{(i)}\right)$ is an associated weight vector with OWA, and satisfying $\sum_{j=1}^{k_{i}} w_{j}^{(i)}=1$, $w_{j}^{(i)}>0 .\left(\sigma_{(1)}, \sigma_{(2)}, \cdots, \sigma_{\left(k_{i}\right)}\right)$ is a permutation of $\left(1,2, \cdots, k_{i}\right)$, such that $\tilde{a}_{\sigma_{(j-1)}}^{(i)} \geq \tilde{a}_{\sigma_{(j)}}^{(i)}$ for all $j=1,2, \cdots, n$, then ITFDOWA is called the intuitionistic trapezoidal fuzzy density ordered weighted average operator.

Further, according to the operational rules of intuitionistic trapezoidal fuzzy numbers, we can get: 


$$
\begin{gathered}
\operatorname{ITFDOWA}\left(\tilde{a}_{1}, \tilde{a}_{2}, \cdots, \tilde{a}_{n}\right)= \\
\left(\left[\sum_{i=1}^{m} \xi_{i} \sum_{j=1}^{k_{i}} w_{j}^{(i)} a_{\sigma(j)}^{(i)}, \sum_{i=1}^{m} \xi_{i} \sum_{j=1}^{k_{i}} w_{j}^{(i)} b_{\sigma(j)}^{(i)}, \sum_{i=1}^{m} \xi_{i} \sum_{j=1}^{k_{i}} w_{j}^{(i)} c_{\sigma(j)}^{(i)}, \sum_{i=1}^{m} \xi_{i} \sum_{j=1}^{k_{i}} w_{j}^{(i)} d_{\sigma(j)}^{(i)}\right] ;\right. \\
\left.1-\prod_{i=1}^{m}\left(1-\left(1-\prod_{j=1}^{k_{i}}\left(1-\mu_{\tilde{a}_{\sigma(j)}^{(i)}}\right)^{w_{j}^{(i)}}\right)\right)^{\xi_{i}}, \prod_{i=1}^{m}\left(\prod_{j=1}^{k_{i}}\left(v_{\tilde{a}_{\sigma(j)}^{(i)}}\right)^{w_{j}^{(i)}}\right)^{\xi_{i}}\right) .
\end{gathered}
$$

From Definitions 13 and 14, we know that the ITFDWA operator weights the intuitionistic trapezoidal fuzzy numbers while the ITFDOWA operator weights the ordered positions of the intuitionistic trapezoidal fuzzy numbers instead of weighting the arguments themselves. Both the operators consider only one aspect. To overcome this drawback, in the following we shall propose an intuitionistic trapezoidal fuzzy density hybrid weighted average (ITFDHWA ) operator.

Definition 15: Let $\tilde{a}_{j}=\left(\left[a_{j}, b_{j}, c_{j}, d_{j}\right] ; \mu_{j}, v_{j}\right)$ be a collection of intuitionistic trapezoidal fuzzy numbers, and $\omega=\left(\omega_{1}, \omega_{2}, \cdots, \omega_{n}\right)^{T}$ be the weight vector of $\tilde{a}_{j}(j=1,2, \cdots, n)$, where $\omega_{j} \geq 0, j=1,2, \cdots, n$ and $\sum_{j=1}^{n} \omega_{j}=1$, then we can define an intuitionistic trapezoidal fuzzy density hybrid weighted average ( ITFDHWA ) operator as follows:

$$
\operatorname{ITFDHWA}\left(\tilde{a}_{1}, \tilde{a}_{2}, \cdots, \tilde{a}_{n}\right)=\sum_{i=1}^{m} \xi_{i} \bullet \operatorname{HWA}\left(\mathrm{A}_{\mathrm{i}}\right)=\sum_{i=1}^{m} \xi_{i}\left(\sum_{j=1}^{k_{i}} w_{j}^{(i)} \tilde{a}_{\sigma(j)}^{(i)}\right)
$$

where: $\mathrm{A}_{i}=\left\{\tilde{a}_{j}^{(i)} \mid i=1,2, \cdots, m ; j=1,2, \cdots, k_{i}\right\}$, and $\sum_{i=1}^{m} k_{i}=n . \mathrm{A}_{1}, \mathrm{~A}_{2}, \cdots, \mathrm{A}_{\mathrm{m}}$ are $m$ clustering classes, and $\tilde{a}_{j}^{(i)}$ is the data of $\mathrm{A}_{\mathrm{i}} ; \xi=\left(\xi_{1}, \xi_{2}, \cdots, \xi_{m}\right)$ is the density weighted vector, and $w_{i}=\left(w_{1}^{(i)}, w_{2}^{(i)}, \ldots, w_{k_{i}}^{(i)}\right)$ is an associated weight vector with OWA , and satisfying $\sum_{j=1}^{k_{i}} w_{j}^{(i)}=1$, $w_{j}^{(i)}>0 .\left(\sigma_{(1)}, \sigma_{(2)}, \cdots, \sigma_{\left(k_{i}\right)}\right)$ is a permutation of $\left(1,2, \cdots, k_{i}\right)$, such that $\tilde{a}_{\sigma_{(j-1)}^{\prime(i)}} \geq \tilde{a}_{\sigma_{(j)}^{\prime}}^{\prime(i)}$ $\left(\tilde{a}_{j}^{(i)}=n \omega_{j}^{(i)} \tilde{a}_{j}^{(i)}\right)$ for all $j=1,2, \cdots, n$, then ITFDHWA is called the intuitionistic trapezoidal fuzzy density hybrid weighted average operator.

Further, according to the operational rules of intuitionistic trapezoidal fuzzy numbers, we can get:

$$
\begin{gathered}
\operatorname{ITFDHWA}\left(\tilde{a}_{1}, \tilde{a}_{2}, \cdots, \tilde{a}_{n}\right)= \\
\left(\left[\sum_{i=1}^{m} \xi_{i} \sum_{j=1}^{k_{i}} w_{j}^{(i)} a_{\sigma(j)}^{\prime(i)}, \sum_{i=1}^{m} \xi_{i} \sum_{j=1}^{k_{i}} w_{j}^{(i)} b_{\sigma(j)}^{\prime(i)}, \sum_{i=1}^{m} \xi_{i} \sum_{j=1}^{k_{i}} w_{j}^{(i)} c_{\sigma(j)}^{(i)}, \sum_{i=1}^{m} \xi_{i} \sum_{j=1}^{k_{i}} w_{j}^{(i)} d_{\sigma(j)}^{\prime(i)}\right] ;\right. \\
\left.1-\prod_{i=1}^{m}\left(1-\left(1-\prod_{j=1}^{k_{i}}\left(1-\mu_{\tilde{a}_{\sigma(j)}^{\prime(i)}}\right)^{w_{j}^{(i)}}\right)\right)^{\xi_{i}}, \prod_{i=1}^{m}\left(\prod_{j=1}^{k_{i}}\left(v_{\tilde{a}_{\sigma(j)}^{\prime(i)}}\right)^{w_{j}^{(i)}}\right)^{\xi_{i}}\right) .
\end{gathered}
$$




\section{Multi-attribute decision making methods based on intuitionistic trapezoidal fuzzy numbers}

Consider a multiple attribute decision making problem with intuitionistic trapezoidal fuzzy numbers: let $A=\left\{A_{1}, A_{2}, \cdots, A_{m}\right\}$ be a discrete set of alternatives, and $C=\left\{C_{1}, C_{2}, \cdots, C_{n}\right\}$ be the set of attributes, $\omega=\left(\omega_{1}, \omega_{2}, \cdots, \omega_{n}\right)^{T}$ is the weighting vector of the attribute $C_{j}(j=1,2, \cdots, n)$, where $\omega_{j} \geq 0, j=1,2, \cdots, n, \sum_{j=1}^{n} \omega_{j}=1$. Suppose that $\tilde{R}=\left[\tilde{r}_{i j}\right]_{m \times n}$ is the decision matrix, where $\tilde{r}_{i j}=\left(\left[a_{i j}, b_{i j}, c_{i j}, d_{i j}\right] ; \mu_{i j}, v_{i j}\right)$ takes the form of the intuitionistic trapezoidal fuzzy number for alternative $A_{i}$ with respect to attribute $C_{j}$. Then, the ranking of alternatives is required.

In the following, we will propose a multiple attribute decision making method based on the density aggregation operators. We firstly convert the decision matrix in intuitionistic trapezoidal fuzzy numbers to interval numbers, and then we use the interval density aggregation operators to derive the overall preference values in interval numbers. Finally, we can rank all alternatives by sorting the interval numbers. The method involves the following steps:

Step 1: Calculate the expected value intervals for all attribute values by Eqs. (10) and (12), we can get:

$$
\tilde{x}_{i j}=\left[I_{\lambda}^{L}\left(\tilde{r}_{i j}\right), I_{\lambda}^{R}\left(\tilde{r}_{i j}\right)\right] .
$$

Step 2: Standardize the expected value intervals, we can obtain the normalized value $\bar{x}_{i j}=\left[\bar{x}_{i j}^{L}, \bar{x}_{i j}^{R}\right]$ by the method of vector transformation.

$$
\left\{\begin{array}{l}
\bar{x}_{i j}^{L}=I_{\lambda}^{L}\left(\tilde{r}_{i j}\right) / \sqrt{\sum_{i=1}^{m}\left(\left(I_{\lambda}^{L}\left(\tilde{r}_{i j}\right)\right)^{2}+\left(I_{\lambda}^{R}\left(\tilde{r}_{i j}\right)\right)^{2}\right)} \\
\bar{x}_{i j}^{R}=I_{\lambda}^{R}\left(\tilde{r}_{i j}\right) / \sqrt{\sum_{i=1}^{m}\left(\left(I_{\lambda}^{L}\left(\tilde{r}_{i j}\right)\right)^{2}+\left(I_{\lambda}^{R}\left(\tilde{r}_{i j}\right)\right)^{2}\right)}
\end{array} \quad(1 \leq i \leq m, 1 \leq j \leq n) .\right.
$$

Step 3: Cluster the expected value intervals by Eqs. (13)-(16).

Step 4: Calculate the density weighted vector by Eqs. (17)-(29).

Step 5: Apply the IDWA operator (or IDOWA, IDHWA operators) to derive the overall preference values in interval numbers.

Step 6: Rank all alternatives by sorting the interval numbers.

Step 7: end.

\section{An illustrative example}

In this section, we use the proposed methods to analysis an example which is used in Wang and Zhang (2008). An Engine part manufacturing company wants to selects the best suppliers according to their core competencies. Suppose that there are five suppliers $\left(a_{1}, a_{2}, a_{3}, a_{4}, a_{5}\right)$ whose core competencies are evaluated by the following five criterions $(\mathrm{C} 1, \mathrm{C} 2, \mathrm{C} 3, \mathrm{C} 4, \mathrm{C} 5)$ : the capability of supplying $(\mathrm{C} 1)$, the capability of delivery $(\mathrm{C} 2)$, the quality of service $(\mathrm{C} 3)$, 
the capability of influence (C4), the strength of scientific research (C5). The criteria weight is $\omega^{*}=(0.15,0.30,0.10,0.15,0.30)$. Decision makers give the evaluation information for all suppliers with respect to all criterions which are listed in Table 1.

Table 1. Criteria values for all alternatives

\begin{tabular}{cccccc}
\hline & $C 1$ & $C 2$ & $C 3$ & $C 4$ & $C 5$ \\
\hline$a_{1}$ & $([1,2,3,4] ; 0.7,0.3)$ & $([5,6,7,8] ; 0.7,0.3)$ & $([3,4,5,6] ; 0.6,0.2)$ & $([4,5,7,8] ; 0.6,0.3)$ & $([4,5,6,7] ; 0.8,0)$ \\
$a_{2}$ & $([2,3,4,5] ; 0.6,0.3)$ & $([6,7,8,9] ; 0.8,0.1)$ & $([4,5,6,7] ; 0.8,0.2)$ & $([3,4,5,6] ; 0.7,0.3)$ & $([6,7,8,9] ; 0.6,0.3)$ \\
$a_{3}$ & $([1,2,3,5] ; 0.6,0.4)$ & $([4,6,7,8] ; 0.6,0.3)$ & $([3,4,5,6] ; 0.5,0.5)$ & $([4,5,6,7] ; 0.8,0.1)$ & $([5,6,7,8] ; 0.8,0.2)$ \\
$a_{4}$ & $([2,3,4,6] ; 0.6,0.2)$ & $([5,6,7,8] ; 0.8,0.2)$ & $([2,3,5,6] ; 0.6,0.4)$ & $([3,4,5,7] ; 0.6,0.3)$ & $([4,6,7,8] ; 0.6,0.3)$ \\
$a_{5}$ & $([2,3,4,5] ; 0.8,0.2)$ & $([4,5,6,7] ; 0.9,0)$ & $([3,4,5,6] ; 0.8,0.2)$ & $([3,5,7,8] ; 0.7,0.1)$ & $([4,5,6,7] ; 0.8,0)$ \\
\hline
\end{tabular}

\subsection{The decision steps for this example}

(1) Calculate the expected values for intuitionistic trapezoidal fuzzy numbers by Eqs. (10) and (12) which are listed in Table 2. (Supposed that the decision makers are indifferent to the risk, and then $\lambda=0.5$ )

Table 2. The expected values of all criteria values

\begin{tabular}{cccccc}
\hline & $C 1$ & $C 2$ & $C 3$ & $C 4$ & $C 5$ \\
\hline$a_{1}$ & {$[1.75,1.75]$} & {$[4.55,4.55]$} & {$[2.70,3.60]$} & {$[3.60,4.20]$} & {$[4.40,5.50]$} \\
$a_{2}$ & {$[2.10,2.45]$} & {$[6.00,6.75]$} & {$[4.40,4.40]$} & {$[3.15,3.15]$} & {$[4.50,5.25]$} \\
$a_{3}$ & {$[1.65,1.65]$} & {$[3.75,4.38]$} & {$[2.25,2.25]$} & {$[4.40,4.95]$} & {$[5.20,5.20]$} \\
$a_{4}$ & {$[2.25,3.00]$} & {$[5.20,5.20]$} & {$[2.40,2.40]$} & {$[2.85,3.33]$} & {$[3.75,4.38]$} \\
$a_{5}$ & {$[2.80,2.80]$} & {$[4.95,5.50]$} & {$[3.60,3.60]$} & {$[4.03,5.18]$} & {$[4.40,5.50]$} \\
\hline
\end{tabular}

(2) Standardize the expected value intervals which are listed in Table 3.

Table 3. The standardization of the expected values

\begin{tabular}{cccccc}
\hline & $\mathrm{C} 1$ & $\mathrm{C} 2$ & $\mathrm{C} 3$ & $\mathrm{C} 4$ & $\mathrm{C} 5$ \\
\hline$a_{1}$ & {$[0.2433,0.2433]$} & {$[0.2795,0.2795]$} & {$[0.2161,0.3488]$} & {$[0.2878,0.3358]$} & {$[0.2874,0.3593]$} \\
$a_{2}$ & {$[0.2920,0.3407]$} & {$[0.3686,0.4146]$} & {$[0.4263,0.4263]$} & {$[0.2518,0.2518]$} & {$[0.2490 .0 .3430]$} \\
$a_{3}$ & {$[0.2294,0.2294]$} & {$[0.2304,0.2688]$} & {$[0.2180,0.2180]$} & {$[0.3518,0.3957]$} & {$[0.3397,0.3397]$} \\
$a_{4}$ & {$[0.3128,0.4171]$} & {$[0.3194,0.3194]$} & {$[0.2325 .0 .2325]$} & {$[0.2278,0.2658]$} & {$[0.2450,0.2858]$} \\
$a_{5}$ & {$[0.3893,0.3893]$} & {$[0.3041,0.3379]$} & {$[0.3488,0.3488]$} & {$[0.3218,0.4137]$} & {$[0.2874,0.3593]$} \\
\hline
\end{tabular}

(3) Cluster the expected value intervals by the method proposed by Yu and Fan (2003), we can get:

$$
A_{1}=\left\{C_{2}, C_{4}, C_{5}\right\}, A_{2}=\left\{C_{1}\right\}, A_{3}=\left\{C_{3}\right\}
$$


(4) Calculate the density weighted vector by Eqs. (17)-(29).

(i) Let the density weighted vector be $\xi=\left(\xi_{1}, \xi_{2}, \xi_{3}\right)$, and then:

$$
\begin{aligned}
& \xi_{1}=\rho \omega_{1}^{s}+0.75 \mathrm{v} ; \\
& \xi_{2}=\rho \omega_{2}^{s}+0.15 \mathrm{v} ; \\
& \xi_{3}=\rho \omega_{3}^{s}+0.10 \mathrm{v} .
\end{aligned}
$$

(ii) Set scale gain function to $f^{+}(\gamma)=a+b e^{(c \gamma)}, \gamma=k_{i} / n, \mathrm{c}=1$, and based on the three properties of the function, we can get the following equations:

$$
\left\{\begin{array}{l}
a+b=0 \\
3 a+2 b e^{0 . .2}+b e^{0.6}=1
\end{array} .\right.
$$

Then we can get $a=-0.79, b=0.79$. So, the scale gain function can be expressed as $f^{+}(\gamma)=-0.79+0.79 e^{\gamma}$.

Based on $\omega_{i}^{s}=\omega_{i}^{s}\left(k_{i} / n\right)=f^{ \pm}\left(k_{i} / n\right)$, we can get $\omega_{1}^{s}=f^{+}(0.6)=0.64, \omega_{2}^{s}=\omega_{3}^{s}=$ $f^{+}(0.2)=0.18$.

(iii) Calculate the values of $\rho$ and $v$.

We can set $\pi=0.8$, then based on Eq. (26), we can get $\rho=\frac{5}{19}, v=\frac{14}{19}$.

(iv) Calculate density weighted vector by (17), then $\xi=\left(\xi_{1}, \xi_{2}, \xi_{3}\right)=(0.72,0.16,0.12)$.

(5) Apply the IDWA operator (or IDOWA, IDHWA operators) to derive the overall preference value in interval numbers.

For the alternative $a_{1}$, we can get:

$$
\begin{gathered}
a_{1}=\mathrm{IDWA}=0.72 \times A_{1}+0.16 \times A_{2}+0.12 \times A_{3}= \\
0.72 \times\{[0.2795,0.2795] \times 0.3+[0.2878,0.3358] \times 0.15+[0.2874,0.3593] \times 0.3\}+ \\
0.16 \times[0.2433,0.2433] \times 0.15+0.12 \times[0.2616,0.3488] \times 0.1=[0.1625,0.1843] .
\end{gathered}
$$

Similarly, we can get the overall preference values of the other alternatives $a_{2}, a_{3}, a_{4}, a_{5}$ which are shown as follows:

$a_{2}=[0.1824,0.2041], a_{3}=[0.1692,0.1823], a_{4}=[0.1568,0.1722], a_{5}=[0.1760,0.2088]$.

(6) Rank all alternatives by sorting the interval numbers.

According to the probably degree method which is proposed by $\mathrm{Xu}$ and $\mathrm{Da}$ (2003), we $\operatorname{rank} a_{1}, a_{2}, a_{3}, a_{4}, a_{5}$, and get that:

$$
a_{2} \succ a_{5} \succ a_{3} \succ a_{1} \succ a_{4}
$$

\subsection{Comparison with the existing methods}

In order to verify the validity of the proposed method, we use the extended VIKOR method proposed by Du and Liu (2011), the grey relational projection method proposed by Zhang et al. (2013), the methods based on the intuitionistic trapezoidal fuzzy weighted arithmetic 
averaging operator and weighted geometric averaging operator proposed by Wang and Zhang (2009b) to the above example, and they can get the ranking result: $a_{2} \succ a_{5} \succ a_{1} \succ a_{3} \succ a_{4}$. Of course, they are the same as the result in Wang and Zhang (2008). Comparing with these methods, the proposed method in this paper can consider the distribution of density degree of the attribute values, and the attribute values can be weighted according to the density of decision making information. In addition, comparing with the methods proposed by $\mathrm{Du}$ and Liu (2011), Zhang et al. (2013), Wang and Zhang (2008), the proposed method not only provides a ranking of the alternatives, but also provides the overall preference values for all alternatives, However, these existing methods can only provide the ranking of the alternatives. Comparing with the methods proposed by Wang and Zhang (2009b), the proposed method is the extensions of the methods proposed by Wang and Zhang (2009b), and when the density weights are equal in each clustering class, the ITFDWA operator can reduce to ITFWA operator which was proposed by Wang and Zhang (2009b). So, the proposed method has more advantages than the existing methods.

\section{Conclusions}

In this paper, with respect to multiple attribute decision making (MADM) problems in which the attribute value takes the form of intuitionistic trapezoidal fuzzy number, some new decision making analysis methods are developed. Firstly, some operational laws and expected values of intuitionistic trapezoidal fuzzy numbers are introduced, and the comparison method for the intuitionistic trapezoidal fuzzy numbers is proposed. Then, the method of calculating density weighted vector has been discussed in detail, and some density aggregation operators based on interval numbers and intuitionistic trapezoidal fuzzy numbers are developed, and a multiple attribute decision making method is presented. The characteristics of these methods are that the density level of the information distribution is considered to weight the attribute values. Finally, an illustrative example is given to illustrate the decision-making steps, to verify the developed methods and to demonstrate its practicality and effectiveness. In the future, we shall continue working in the extension and application of the developed method to other domains, and extension of ITFDWA, ITFDOWA and ITFDHWA operators to the multiple attribute decision making.

\section{Acknowledgment}

This paper is supported by the National Natural Science Foundation of China (No. 71271124), the Humanities and Social Sciences Research Project of Ministry of Education of China (No. 13YJC630104), the Natural Science Foundation of Shandong Province (No. ZR2011FM036), Shandong Provincial Social Science Planning Project (No.13BGLJ10), and graduate education innovation projects in Shandong Province (SDYY12065). The author also would like to express appreciations to the anonymous reviewers and Editor in Editor for their very helpful comments that improved the paper. 


\section{References}

Atanassov, K. T. 1986. Intuitionistic fuzzy sets, Fuzzy Sets and Systems 20(1): 87-96. http://dx.doi.org/10.1016/S0165-0114(86)80034-3

Atanassov, K. T. 1989. More on intuitionistic fuzzy sets, Fuzzy Sets and Systems 33(1): 37-46. http://dx.doi.org/10.1016/0165-0114(89)90215-7

Du, Y.; Liu, P. D. 2011. Extended fuzzy VIKOR method with intuitionistic trapezoidal fuzzy numbers, Information - An International Interdisciplinary Journal 14(8): 2575-2584.

Hou, F.; Guo Y. J. 2008. Interval number density middle operator in uncertain multiple attribute decision making, Journal of Northeastern University (Natural Science) 29(10): 1509-1516.

Li, W. W.; Yi P. T.; Guo Y. J. 2012. Interval number density operator and its application, Journal of Northeastern University (Natural Science) 33(7): 1043-1046.

Liu, P. D. 2009. Multi-attribute decision-making method research based on interval vague set and TOPSIS method, Technological and Economic Development of Economy 15(3): 453-463.

Liu, P. D. 2013a. Some generalized dependent aggregation operators with intuitionistic linguistic numbers and their application to group decision making, Journal of Computer and System Sciences 79(1): 131-143. http://dx.doi.org/10.1016/j.jcss.2012.07.001

Liu, P. D. 2013b. The multi-attribute group decision making method based on the interval grey linguistic variables weighted aggregation operator, Journal of Intelligent and Fuzzy Systems 24(2): 405-414.

Liu, P. D. 2013c. Some Hamacher aggregation operators based on the interval-valued intuitionistic fuzzy numbers and their application to group decision making, IEEE Transactions on Fuzzy systems PP(99): 1. http://dx.doi.org/10.1109/TFUZZ.2013.2248736

Liu, P. D.; Jin, F. 2012. Methods for aggregating intuitionistic uncertain linguistic variables and their application to group decision making, Information Sciences 205: 58-71.

http://dx.doi.org/10.1016/j.ins.2012.04.014

Liu, P. D.; Zhang, X.; Fang Jin, F. 2012. A multi-attribute group decision-making method based on interval-valued trapezoidal fuzzy numbers hybrid harmonic averaging operators, Journal of Intelligent \& Fuzzy Systems 23(5): 159-168.

Razavi Hajiagha, S. H.; Hashemi, S. S.; Zavadskas, E. K. 2013. A complex proportional assessment method for group decision making in an interval-valued intuitionistic fuzzy environment, Technological and Economic Development of Economy 19(1): 22-37. http://dx.doi.org/10.3846/20294913.2012.762953

Wan, S. P. 2013. Power average operators of trapezoidal intuitionistic fuzzy numbers and application to multi-attribute group decision making, Applied Mathematical Modelling 37(6): 4112-4126. http://dx.doi.org/10.1016/j.apm.2012.09.017

Wang, J. Q. 2008. Overview on fuzzy multi-criteria decision-making approach, Control and Decision 23: 601-606.

Wang, J. Q.; Zhang, Z. H. 2008. Programming method of multi-criteria decision-making based on intuitionistic fuzzy number with incomplete certain information, Control and Decision 23: 1145-1148.

Wang, J. Q.; Zhang, Z. H. 2009a. Multi-criteria decision-making method with incomplete certain information based on intuitionistic fuzzy number, Control and Decision 24: 226-230.

Wang, J. Q.; Zhang, Z. H. 2009b. Aggregation operators on intuitionistic trapezoidal fuzzy number and its application to multi-criteria decision making problems, Journal of Systems Engineering and Electronics 20: 321-326.

Xu, Z. S. 2007. Intuitionistic fuzzy aggregation operators, IEEE Transactions on Fuzzy Systems 15(6): 1179-1187. http://dx.doi.org/10.1109/TFUZZ.2006.890678

Xu, Z. S.; Da Q. L. 2003. Possibility degree method for ranking interval numbers and its application, Journal of Systems Engineering 18(1): 67-70. 
Xu, Z. S.; Yager, R. R. 2006. Some geometric aggregation operators based on intuitionistic fuzzy sets, International Journal of General Systems 35(4): 417-433. http://dx.doi.org/10.1080/03081070600574353

Yi, P. T.; Guo, Y. J. 2010. Generalized real density weighted averaging middle operator and its application, Journal of Systems Engineering 25(2): 196-202.

Yi, P. T.; Guo, Y. J.; Zhang, D. N. 2007. Density weighted averaging middle operator and application in multi-attribute decision making, Control and Decision 22: 515-524.

Yu, C. H.; Fan, Z. P. 2003. A clustering method forinterval numberinformation based on risk attitude factor, Journal of Northeastern University (Natural Science) 11(24): 1116-1118.

Yu, D. J. 2013. Intuitionistic fuzzy prioritized operators and their application in multi-criteria group decision making, Technological and Economic Development of Economy 19(1): 1-21. http://dx.doi.org/10.3846/20294913.2012.762951

Zhang, X.; Jin, F.; Liu, P. D. 2013. A grey relational projection method for multi-attribute decision making based on intuitionistic trapezoidal fuzzy number, Applied Mathematical Modelling 37(5):3467-3477. http://dx.doi.org/10.1016/j.apm.2012.08.012

Zhang, X.; Liu, P. D. 2010. Method for aggregating triangular fuzzy intuitionistic fuzzy information and its application to decision making, Technological and Economic Development of Economy 16(2): 280-290. http://dx.doi.org/10.3846/tede.2010.18

Peide LIU obtained his Doctoral degree in Management Science and Engineering in the Beijing Jiaotong University, obtained his Master's degree in Signal and Information Processing in the Southeast University, and obtained his Bachelor's degree in Signal and Information Processing in the Southeast University. Now, he is currently a Professor with School of Management Science and Engineering in Shandong University of Finance and Economics, and he is an Associate Editor of Journal of Intelligent and Fuzzy Systems, a member of Editorial Board of Technological and Economic Development of Economy, The Scientific World Journal, etc. He has authored or coauthored more than 100 publications. His main research fields are decision analysis and decision support, applied mathematics, expert systems, technology and information management, intelligent information processing.

Xiaocun YU is studying for a Master's degree in Information Management with School of Management Science and Engineering in Shandong University of Finance and Economics. Her main research fields are decision analysis and decision support, electronic-commerce, etc. 